\title{
Photophysics and electroluminescence of single nanocrystals of halide perovskites and related nanomaterials
}

\author{
Martin Vacha*, and Dharmendar Kumar Sharma \\ Department of Materials Science and Engineering, Tokyo Institute of Technology, Ookayama 2-12-1, \\ Meguro-ku, Tokyo 152-8552, Japan
}

\begin{abstract}
We report simultaneous photoluminescence and electroluminescence single-particle study of nanocrystals of inorganic halide perovskite $\mathrm{CsPbBr}_{3}$, as well as of ternary I-III-IV semiconductor quantum dots.
\end{abstract}

Due to their exceptional excitonic properties such as strong absorption and efficient narrow-band emission, lead halide perovskite nanocrystals (NCs) have been recently rediscovered as possible alternatives to semiconductor quantum dots for electroluminescence (EL) devices and other light-emitting and sensing applications, and research into their basic photophysical properties is attracting increasing attention. While dispersions of NCs generally show high photoluminescence (PL) quantum yields, the efficiencies of electrically driven luminescence devices are considerably lower. We have shown previously that simultaneous characterization of PL and EL on single-molecule level can bring valuable insight into these emission processes in conjugated polymers [1] and small molecules [2]. Especially, the differences in luminescence blinking and spectral dynamics between the two processes can provide information on the different dynamics of the excited states and their relaxations. Here, we report a simultaneous study of EL and PL of inorganic halide perovskite nanocrystals with the aim to uncover the origin and ways of suppression of blinking in both EL and PL to maximize the emission quantum yield, as well as to study the origin of spectral diffusion and spectral shifts, in relationship with structural/compositional stability.

We use nanocrystals of $\mathrm{Cs} \mathrm{PbBr}_{3}$ prepared by methods reported in literature. In solution, the NCs show efficient and narrow emission in the green part of the spectrum. We first examined the influence of a surrounding matrix on their spectral properties and blinking behavior by dispersing the NCs in an inert matrix of PMMA and in a conductive matrix of $\mathrm{PVK} / \mathrm{PBD}$, both on the level of single NCs and aggregates (arrays) of tens of NCs. The differences in the blinking characteristics appear as different parameters of truncated power law distributions of the on-times, both for bright and dim (grey) states. These findings are further corroborated by an effect of externally applied electric field on the blinking dynamics and PL spectra. The applied field enhances the blinking in both matrices, and

\footnotetext{
* Corresponding author: vacha.m.aa@m.titech.ac.jp
} 
causes a quadratic Stark shift, the extent of which differs from particle to particle. Based on the above characterization, we next fabricate a single-particle organic light-emitting device (OLED) by dispersing the NCs at very low concentrations in PVK/PBD matrix as an emitting layer in an ITO/PEDOT:PSS/PVK/PBD/TPBi/LiF/Al device and study the device by PL and EL single-particle spectroscopy. As shown in an example in the Figure 1, the same NCs can exhibit dramatically different brightness and blinking behavior upon the different modes of excitations. Such differences in PL and EL characteristics can be attributed partly to influence of charge injection and trapping in the device, but intrinsic properties of the NC arrays also play a role in the observed phenomena.
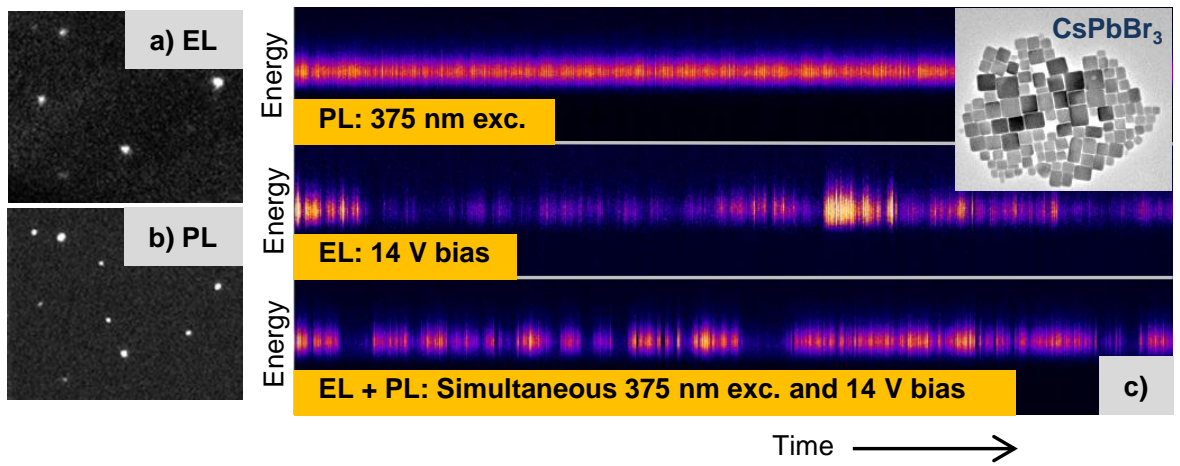

Fig. 1. Electroluminecsnece (a) and photoluminescence (b) microscopic images of $\mathrm{CsPbBr}_{3}$ nanocrystals in an ITO/PEDOT:PSS/PVK/PBD/TPBi/LiF/Al device. (c) Spectral time traces measured from one bright spot in the images upon excitiation by light, electric field and combined show different blinking and lineshape dynamics.

Apart from the work on perovskite nanocrystals, we report on recent progress in singleparticle photophysical characterization of ternary I-III-IV semiconductor nanocrystals which have been explored as a non-toxic alternative to II-VI semiconductor quantum dots. For NCs of $(\mathrm{AgIn})_{x} \mathrm{Zn}_{2(1-x)} \mathrm{S}_{2}$ we explore the origin of defect emission, multiple emitting sites and suppression of blinking [3], and report the emergence of narrow band-edge PL in core/shell structures [4].

\section{References}

1. Y. Honmou, S. Hirata, H. Komiyama, J. Hiyoshi, S. Kawauchi, T. Iyoda and M. Vacha, Nature Commun. 5, 4666 (2014)

2. Y. Sekiguchi, S. Habuchi and M. Vacha, ChemPhysChem 10, 1195 (2009)

3. D. K. Sharma, S. Hirata, L. Bujak, V. Biju, T. Kameyama, M. Kishi, T. Torimoto and M. Vacha, Nanoscale 8, 13687 (2016)

4. T. Uematsu, K. Wajima, D. K. Sharma, S. Hirata, T. Yamamoto, T. Kameyama, M. Vacha, T. Torimoto, S. Kuwabata, NPG Asia Mater. DOI : 10.1038/s41427-0180067-9 (2018) 